\title{
A Biobehavioural Approach to Understand How Mindfulness-Based Cognitive Therapy Reduces Dispositional Negative Self-Bias in Recurrent Depression
}

\author{
Hans Kirschner ${ }^{1,2}$ (D) $\cdot$ Willem Kuyken ${ }^{3} \cdot$ Anke Karl $^{2}$
}

Accepted: 15 February 2022 / Published online: 1 March 2022

(c) The Author(s) 2022

\begin{abstract}
Objectives In order to address an important gap in understanding mechanisms of depressive relapse, we complemented selfreport with psychophysiological measures to a specifically developed brief self-compassion exercise, to examine if mindfulness-based cognitive therapy (MBCT) reduces dispositional negative self-bias in individuals with recurrent depression. Methods Individuals' responses (self-report, heart rate, heart rate variability and skin conductance) during a previously validated brief self-compassion exercise were assessed in a repeated-measures experimental design at two timepoints 2.5-3 months apart in a convenience sample of remitted depressed attendees of an outpatient clinic for mood disorders. The first group received eight sessions of $\mathrm{MBCT}(n=25)$ in between the two measurements whereas the second group was an untreated control group $(n=25)$.

Results When the MBCT group engaged in the self-compassion exercise after MBCT, they showed reduced physiological arousal and increased heart rate variability whereas no changes were observed in the untreated control group. Whereas selfreported state self-compassion after the exercise increased in both groups at both timepoints, only in the MBCT group were the overall levels of state and dispositional self-compassion increased significantly.

Conclusion MBCT appears to effectively reduce negative self-bias and facilitates psychophysiological benefits associated with a more positive self-view. Physiological responses to our self-compassion exercise could be useful markers of treatmentrelated changes and further mechanism research on depressive relapse prevention.
\end{abstract}

Keywords Negative self-bias · Depression $\cdot$ MBCT $\cdot$ Self-compassion $\cdot$ Psychophysiology

Worldwide, approximately 350 million people suffer from major depressive disorder (MDD), the most prevalent, chronic mental disorder (Kessler et al., 2009) that is a leading cause of disability (WHO, 2008). With relapse rates of $50-90 \%$, individuals with depression remain highly vulnerable even after symptom reduction (Kessing et al., 2004). Consequently, it is pivotal to study mechanisms driving this vulnerability and to evaluate treatment approaches in their potential to address it effectively.

Hans Kirschner

hans.kirschner@ovgu.de

1 Institute of Psychology, Otto-Von-Guericke University, Magdeburg, Germany

2 School of Psychology, University of Exeter, Exeter, UK

3 Department of Psychiatry, University of Oxford, Oxford, UK
One of the most pervasive and detrimental vulnerability factors of depressive relapse is negative self-bias, i.e. the habitual, highly internalised self-criticism that manifests as self-deprecating thoughts and feelings of worthlessness (American Psychiatric Association, 2013; Williams et al., 1990). It is characterised by hard-to-inhibit access to negative information and difficult-to-retrieve positive information about the self. Although pharmacological and psychological treatments can reduce negative self-bias (Hilimire et al., 2015; Yoshimura et al., 2014, 2017), it persists at an automatic, habitual level beyond symptom reduction and can therefore be easily reactivated (Hedlund \& Rude, 1995; Rude et al., 2002; Segal et al., 2013). This habitual negative self predicts depressive relapse (Disner et al., 2017; LeMoult et al., 2017) and appears difficult to treat (Shestyuk \& Deldin, 2010) making it a particularly hard-to-address key vulnerability factor for depressive relapse (Beck, 2008; Gotlib \& Joormann, 2010; Mathews \& MacLeod, 2005). 
A relatively novel treatment, mindfulness-based cognitive therapy (MBCT), was designed to help people to recognise and decentre from negative self-bias. MBCT is an 8-week psychosocial programme that teaches meditation to cultivate attitudes of acceptance and non-judgement in order to recognise and disengage from unhelpful depressive thinking patterns (Segal et al., 2013). MBCT forms an affordable alternative to long-term anti-depressant medication in preventing depressive relapse (Kuyken et al., 2015, 2016; Piet \& Hougaard, 2011) and has therefore been widely implemented in UK mental health care services (NICE, 2010; Tickell et al., 2020).

Studies directly evaluating MBCT's benefits and mechanisms in reducing negative self-bias in recurrent depression are lacking. Support for a possible role of mindfulness in reducing negative self-bias comes from neuroscience research predominantly conducted in healthy samples and experienced meditators. It suggests that regular practice of moment-to-moment, non-judgemental awareness leads to functional and structural plastic changes in brain circuitries (i.e. insula, medial prefrontal cortex) involved in selfreferential processing (Farb et al., 2007; Tang et al., 2015). Interestingly, these brain circuitries have been altered in depression and are implicated in negative self-bias (Lemogne et al., 2009, 2010).

Additionally, intervention research showed that MBCT, although not explicitly targeting it in the intervention, increases dispositional self-compassion (Holzel et al., 2011; Kuyken et al., 2010). Current definitions of self-compassion were informed by ancient Buddhist philosophy and proposed by researchers who were also practitioners and teachers of compassion meditation and who conducted considerable psychometric research to come forward with a definition that can be applied in psychological research (Feldman \& Kuyken, 2011; Neff, 2003b; Salzberg, 1997; Strauss et al., 2016; Williams et al., 2014). Self-compassion involves being kind to oneself (Neff, 2003b) and meeting adversity with self-reassurance and soothing (Gilbert, 2009; Neff, 2003b). Having compassion also means being non-judgemental about one's self (Gilbert, 2009; Neff, 2003b) and recognising one's experience as part of a shared human experience (Neff, 2003b). Its counterpart self-criticism involves maladaptive emotion-regulation strategies such as judging one's self harshly (Gilbert, 2009; Neff, 2003b). It is further linked to self-isolation (Neff, 2003b) and adopting a flight-or-fight or social rank mode (Gilbert, 2009). Therefore, self-criticism is exacerbating a sense of threat in difficult times.

MBCT-related cultivation of self-compassion also facilitated effective recovery from experimentally induced negative mood in individuals with recurrent depression (Kuyken et al., 2010). Additionally, levels of dispositional self-compassion are associated with more adaptive emotion regulation and more effective repair of negative mood in individuals with current (Diedrich et al., 2014) and remitted depression (Karl et al., 2018). Hence, cultivating selfcompassion could be a key mechanism of change via which MBCT is addressing negative self-bias.

Despite growing evidence for self-compassion's beneficial effects in depression (MacBeth \& Gumley, 2012), selfsoothing can be difficult for people who suffer from recurrent depression (Gilbert et al., 2014). Whereas in healthy populations self-compassion inductions typically lead to an increase in positive self-views (Arch et al., 2014; Kirschner et al., 2019), individuals prone to self-criticism struggle to disengage from negative self and to access self-soothing (Gilbert et al., 2010, 2014). This renders compassion induction exercises useful for assessing individual differences in negative self-bias. Critically, because most self-compassion studies heavily rely on self-report, it is vital to identify mechanisms and indicators more suitable for accessing and targeting the automatic, habitual nature of negative self-bias in order to effectively address depressive relapse.

When asked to engage in a specially developed self-compassion meditation that invites participants to direct kind and self-affirming thoughts first to a significant other and then, for several minutes, to themselves (Kirschner et al., 2019), healthy individuals reported increased state self-compassion that was accompanied by increased heart rate variability (an index assumed to be a measure of parasympathetic stimulation and adaptive emotion regulation (Thayer \& Lane, 2000)) and decreased physiological arousal (indicated by a decreased heart rate and skin conductance levels). In contrast, a self-critical rumination control condition was accompanied by the opposite physiological response pattern indicating a threat response (Kirschner et al., 2019). Similarly, Rockliff et al. (2008) demonstrated that practising compassion was associated with increased heart rate variability and a cortisol decrease. Interestingly, within their sample, high dispositional self-criticism was associated with a decrease in heart rate variability. Based on these findings and because physiological responses underlie far less explicit strategic control, they may be considered a physiological marker of the benefits associated with adopting a positive self. Such a marker may allow us to better differentiate healthy from depressive individuals and inform the potential of therapeutic interventions to facilitate a more positive self.

To the best of our knowledge, it has not been investigated how individuals currently remitted from depression respond to our one-off self-compassion meditation (i.e. if they show evidence for increased self-report (strategic) positive self and the associated physiological response pattern, similarly to healthy individuals). In addition, it has not been experimentally studied if MBCT helps individuals with recurrent depression to reduce negative self-bias and increase positive self-views which could indicate that the latent vulnerability for depressive relapse was effectively addressed. 
We therefore first investigated if prior to MBCT remitted patients with recurrent MDD demonstrated a significant reduction in self-reported state self-criticism and increase in state self-compassion when listening to the induction, to test the assumption that absence of current clinical symptoms is accompanied by absence of explicit negative selfbias (hypothesis 1). Second, we examined if the self-report findings were accompanied by a reduction in physiological arousal and increase in parasympathetic activation when listening to the self-compassion induction as a physiological marker of the benefits of a more positive self (hypothesis 2). Third, our study investigated whether self-report and physiological responses to the brief self-compassion induction improved from pre- to post-MBCT thus investigating if MBCT further reduces negative self-bias and increases positive self (hypothesis 3). Fourth, our study investigated if MBCT-related reductions in physiological arousal and increase in heart rate variability are associated with changes in dispositional self and depressive symptoms (hypothesis 4).

\section{Methods}

\section{Participants}

Fifty individuals who met criteria for recurrent major depressive disorder in full or partial remission according to the DSM-IV (First et al., 1995) were recruited (see Fig. 1 for the participant flow chart). Two groups of individuals were included, (a) those who underwent an 8-week MBCT programme (remitted depressed MBCT group; $n=25$ ) and (b) individuals recruited from the waiting list for an MBCT programme (remitted depressed control group; $n=25$ ). The MBCT course was delivered by a trained MBCT therapist who taught $>10$ courses. The MBCT course strictly followed the manualised programme in length and content (Segal et al., 2013). No changes have been made to the standard MBCT programme to explicitly address compassion or self-compassion. Participants were eligible when 18 years or older, right-handed and native English speakers. They were excluded if they were currently depressed, previously attended MBCT or currently received other psychological therapy. In addition, we screened out participants who (a) had visual or hearing difficulties which were not corrected by contact lenses, glasses or a hearing aid; (b) had very sensitive skin or a diagnosed skin condition; (c) had a history of brain surgery; (d) suffered from high blood pressure; (e) had a change in medication within the last 3 months; (f) had a pacemaker fitted; or (g) suffered from epilepsy. All participants gave written informed consent, and the study was approved by the local Research Ethics Committee. Sample characteristics and group comparisons at baseline are in Table 1.

Sample size was determinate using a priori sample size calculations (Faul et al., 2007). The sample size was determined for a 2 (group) $\times 2$ (time) mixed ANOVA, assuming a statistical power of $0.80, a=0.05$, and a medium effect size $(f=0.25)$. As there were no prior studies with similar designs and measures, we based our power calculations on
Fig. 1 Participant flow diagram. Note: Reason for the exclusion of the physiological dataset was poor data quality. In addition, one questionnaire pack was missing from a participant of the control group

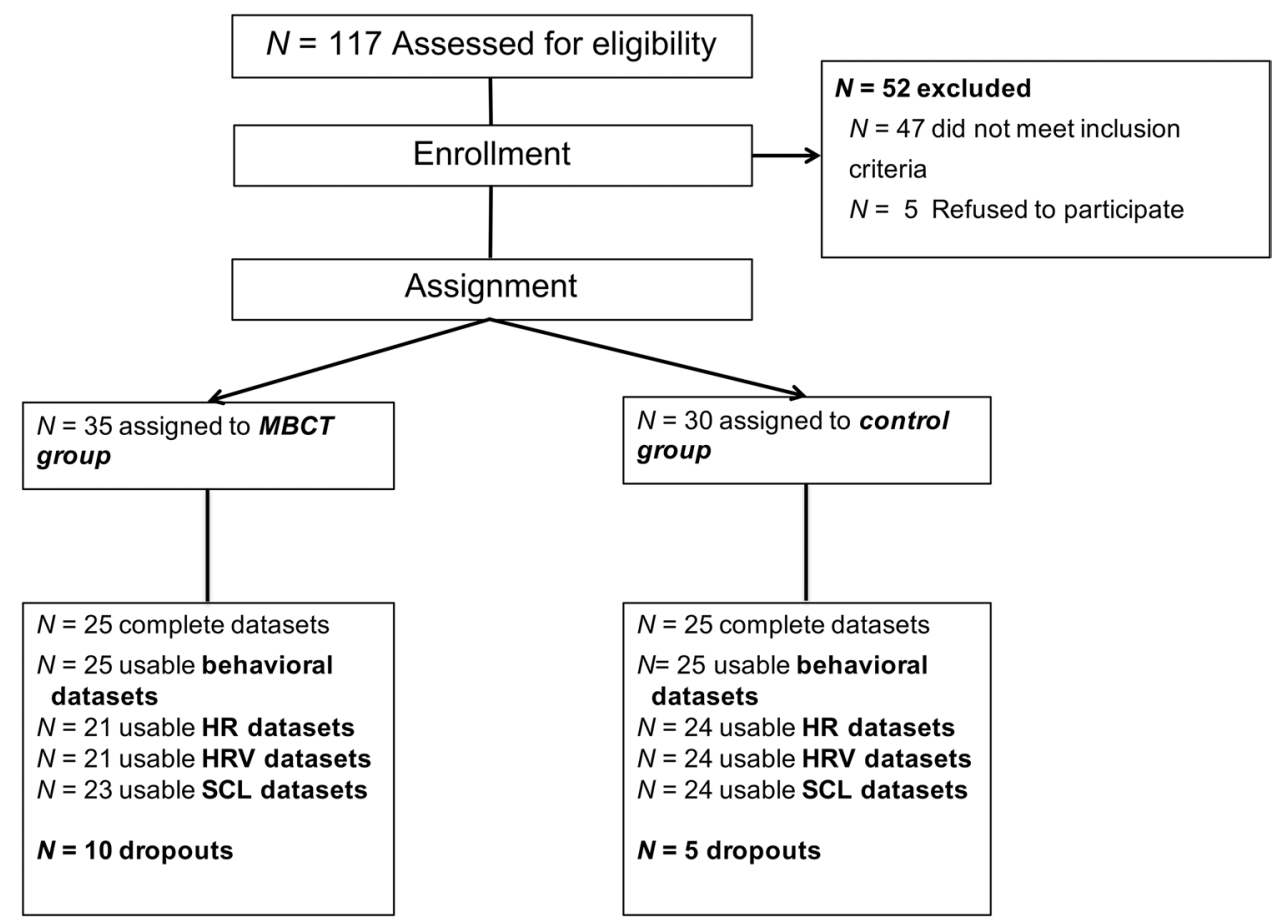


Table 1 Means, standard deviations, and significance tests for the sample characteristics at baseline of the different groups

\begin{tabular}{|c|c|c|c|c|}
\hline \multirow[b]{2}{*}{ Characteristic } & \multicolumn{2}{|l|}{ Baseline } & \multirow[b]{2}{*}{ Test } & \multirow[b]{2}{*}{$p$} \\
\hline & $\begin{array}{l}\text { Remitted } \\
\text { depressed } \\
\text { MBCT }\end{array}$ & $\begin{array}{l}\text { Remitted } \\
\text { depressed } \\
\text { control }\end{array}$ & & \\
\hline$n$ & 25 & 25 & & \\
\hline \multicolumn{5}{|l|}{ Gender } \\
\hline Male/female: $n$ & $10 / 15$ & $5 / 20$ & $\chi^{2}(1, N=50)=2.38$ & .123 \\
\hline Female: \% & 60 & 80 & & \\
\hline Age in years, $M(\mathrm{SD})$ & $49.92(9.68)$ & $47.16(11.89)$ & $t(48)=.90$ & .373 \\
\hline \multicolumn{5}{|l|}{ Material status: $n(\%)$} \\
\hline Single & $6(24)$ & $10(40)$ & & \\
\hline Married or living with someone as if married & $12(48)$ & $12(48)$ & & \\
\hline Separated, divorced, or widowed & $7(28)$ & $3(12)$ & & \\
\hline \multicolumn{5}{|l|}{ Level of education: $n(\%)$} \\
\hline No educational qualification & 0 & 0 & & \\
\hline Some school qualification & 0 & $1(4)$ & & \\
\hline High school and/or vocational qualification & $1(4)$ & $1(4)$ & & \\
\hline University degree/professional qualification & $24(96)$ & $23(92)$ & & \\
\hline Number of depressive episodes: $M$ (SD) & $5.36(4.06)$ & $5.56(5.15)$ & $t(48)=.15$ & .879 \\
\hline Age of onset of first depressive episode: $M$ (SD) & $27.12(8.27)$ & $23.96(8.09)$ & $t(48)=1.37$ & .178 \\
\hline \multicolumn{5}{|l|}{ Medication } \\
\hline Yes/no & $16 / 9$ & $17 / 8$ & $\chi^{2}(1, n=50)=.09$ & .765 \\
\hline Medicated: \% & 64 & 68 & & \\
\hline \multicolumn{5}{|l|}{ Self-compassion scale } \\
\hline Total: $M(\mathrm{SD})$ & $14.58(3.75)$ & $15.91(4.34)$ & $t(47)=1.15$ & .257 \\
\hline \multicolumn{5}{|l|}{ FSCRS } \\
\hline Reassure self: $M(\mathrm{SD})$ & $16.21(3.90)$ & $18.29(5.62)$ & $t(46)=1.49$ & .143 \\
\hline Inadequate self: $M(\mathrm{SD})$ & $20.58(8.62)$ & $15.33(8.61)$ & $t(46)=2.11$ & .040 \\
\hline Hated self: $M(\mathrm{SD})$ & $4.70(3.35)$ & $3.13(3.72)$ & $t(46)=1.55$ & .129 \\
\hline BDI: $M(\mathrm{SD})$ & $16.08(10.21)$ & $11.21(10.07)$ & $t(48)=1.68$ & .100 \\
\hline
\end{tabular}

Note. Trait self-compassion has been assessed via the SCS (Neff, 2003a, 2003b). The possible range of this scale is 0-30, with higher scores indicating higher trait levels of self-compassion. The Forms of Self-Criticising/Attacking \& Self-Reassuring Scale (FSCRS; Gilbert et al., 2004) was used to assess the trait level of self-criticism. The scale measures two forms of self-criticalness, inadequate self (possible range 0-33) and hated self (possible range 0-20), and one form of self-reassure, reassure self (possible range 0-32)

$B D I$ Beck depression inventory a previously published RCT that assessed MBCT-related changes in self-compassion (Kuyken et al., 2010). Based on this calculation, it was found that a minimum of 50 participants were required for this study to detect an effect of group on the outcome variables.

\section{Procedure}

After screening for eligibility, interested participants were invited for a diagnostic assessment where they provided informed consent before we assessed demographic information, history of depression and current depression status through the SCID (First et al., 1995). Participants were then scheduled for the physiology sessions approximately 2-2.5 months apart. The MBCT group was tested immediately before and after the intervention. The control group was tested at similar intervals. The experimental procedure of the physiological sessions is shown in Fig. 2A. At the end of each session, participants completed the questionnaire pack.

\section{Measures}

\section{Experimental Induction}

We used a previously validated, eleven-and-a-half-minute Loving Kindness Meditation for the Self (LKM-S) (Kirschner et al., 2019). The self-compassion manipulation was developed and recorded together with an experienced MBCT therapist. The guided mediation was $11.5 \mathrm{~min}$ long. The 
basis of the manipulation was a Loving Kindness Mediation (LKM; see Salzberg, 1997) that was tailored to specifically cultivate state self-compassion and incorporating the clinical experiences of the therapist. During the manipulation, participants were guided to direct loving/friendly feelings towards a close person. They were then asked to direct the same feelings towards themselves.

\section{Diagnostic Assessment}

We assessed current and past depression through the Structured Clinical Interview for DSM-IV (SCID-IV) (First et al., 1995).

\section{Self-Report Measures}

To assess change processes in MBCT (Feldman \& Kuyken, 2011), trait levels of self-compassion (Self-Compassion Scale (SCS) (Neff, 2003a)), self-criticism (Forms of SelfCriticising/Attacking \& Self-Reassuring Scale (FSCRS) (Gilbert et al., 2004)) and depressive symptom scores (Beck Depression Inventory, Second Edition (BDI-II) (Beck et al., 1996)) were administered pre- and post-treatment. To assess the effectiveness of the self-compassion manipulation on participants' state self-compassion and self-criticism, a series of visual analogue scales (VAS, ranging from 0 to 100) was used throughout the experimental sessions. See Supplemental Materials for more information about the selfreport measures used in this study.

\section{Physiological Measurements}

Physiological responses were recorded using a BIOPAC ${ }^{\text {TM }}$ MP150 system and the software AcqKnowledge 4.2 (BIOPAC Systems; Goleta, CA). Skin conductance level (SCL) as a measure of sympathetic activation and physiological defence response (Sokolov, 1963) was continuously recorded using a BIOPAC SCL100C amplifier and a skinresistant transducer (TSD203) from the middle phalanx of the first and ring fingers of the participant's nondominant hand at a sampling rate of $500 \mathrm{~Hz}$ with a low pass filter of 1.0 Hz. Mean SCL, maximum SCL values, and minimum SCL values were extracted for 1-min segments from baseline to minute 11 of the exercise. A range correction (Lykken et al., 1966) was applied for each participant to give a mean SCL corrected for individual differences. Heart rate (HR) as an indicator of physiological arousal was determined from raw electrocardiogram (ECG) in beats per minute on the basis of a semiautomatic R-wave detection algorithm using AcqKnowledge. Raw ECG was acquired using a BIOPAC ECG100C amplifier at a sampling rate of $1 \mathrm{kHz}$ and filtered using a band pass of $0.5-35 \mathrm{~Hz}$. Artefact detection (i.e. noisy, missing, or ectopic beats) and removal were performed using a template correlation and interpolation from the adjacent R-peaks based on established procedures in less than 5\% of the ECG data (Berntson et al., 1997). Mean HR in beats per minute was then extracted from the $\mathrm{R}$-waves for the same time windows. High-frequency heartrate variability (HF-HRV) as an indicator of parasympathetic activation and adaptive physiological regulation capacity (Thayer \& Lane, 2000) was determined from the artefactfree ECG by submitting a time series of the R-peaks to a fast Fourier transformation that calculated the power spectrum of the R-R interval variation for the frequency range between 0.15 and $0.4 \mathrm{~Hz}$ in a given time window (Berntson et al., 1997). Mean HF HRV were then extracted for each data section similar to the HR. HRV values were transformed using a percent deviation from the mean (Ellis et al., 2008). To obtain SCL, HR and HRV response throughout the audio exercise and in order to control for individual differences, we calculated each participants' change values for each minute of the experimental condition by subtracting the averaged baseline values from the scores during each minute of the audio exercise.

\section{Data Analyses}

\section{Prior Intervention Effects}

Psychophysiological changes in response to the self-compassion exercise were analysed using paired-sample $t$ tests across groups.

\section{Treatment-Related Effects}

Treatment-related changes in depression and dispositional self-compassion and self-criticism were analysed using paired-sample $t$ tests for each experimental group. To test whether MBCT changes psychophysiological responses to the self-compassion exercise, we applied mixed ANOVAs with time (pre- and post-MBCT) as the within-subject factor and group as the between-subject factor. To analyse the physiological response trajectories, we computed an average change score for heart rate, heart rate variability and skin conductance change depending on the focus during the LKM-S (others (min 1-5) vs. self (min 6-11)) for the first and second assessment. For these analyses, focus (others vs. self) was added as an additional within-subject factor to the ANOVAs.

\section{Associations}

In order to study the associations between treatment-related physiological change, change in dispositional self (trait self-compassion and self-criticism) and symptom reduction (changes in BDI scores), we calculated zero-order 
A
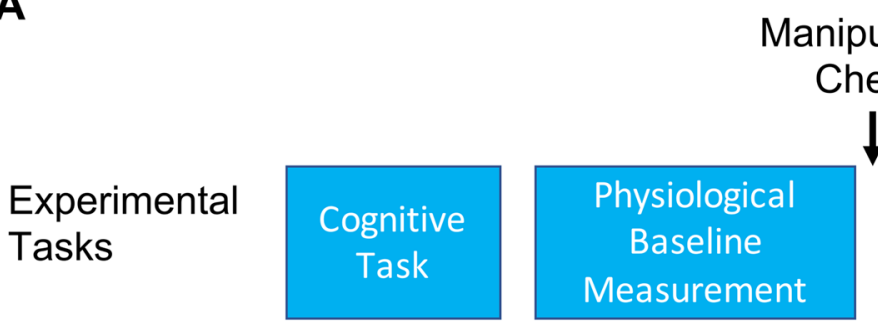

Approximate

Timings

$10 \min$

$8 \mathrm{~min}$

Self-Compassion Exercise

Focus: Other

Focus: Self

$11.5 \min$

$10 \min$

Physiological

EEG

Recordings

\section{ECG \& SCL}

B

C
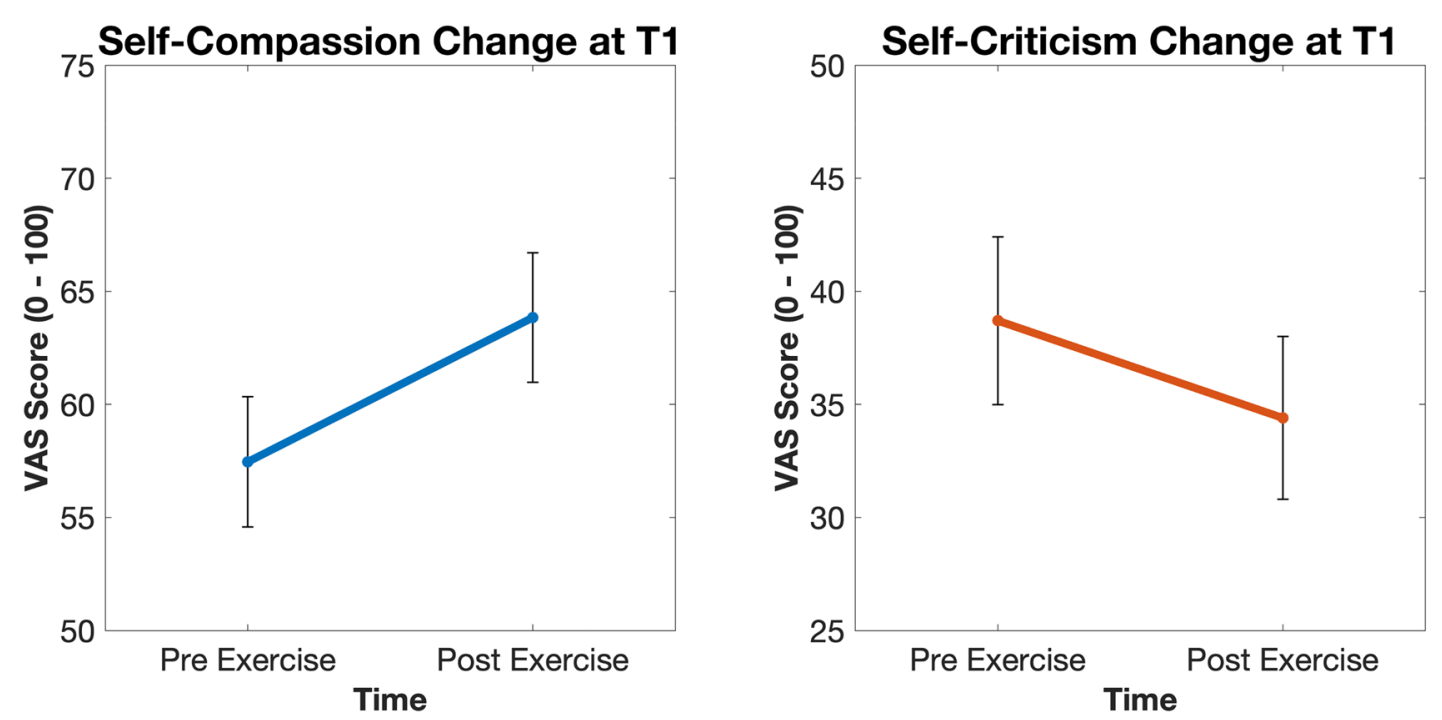

D

E

$F$
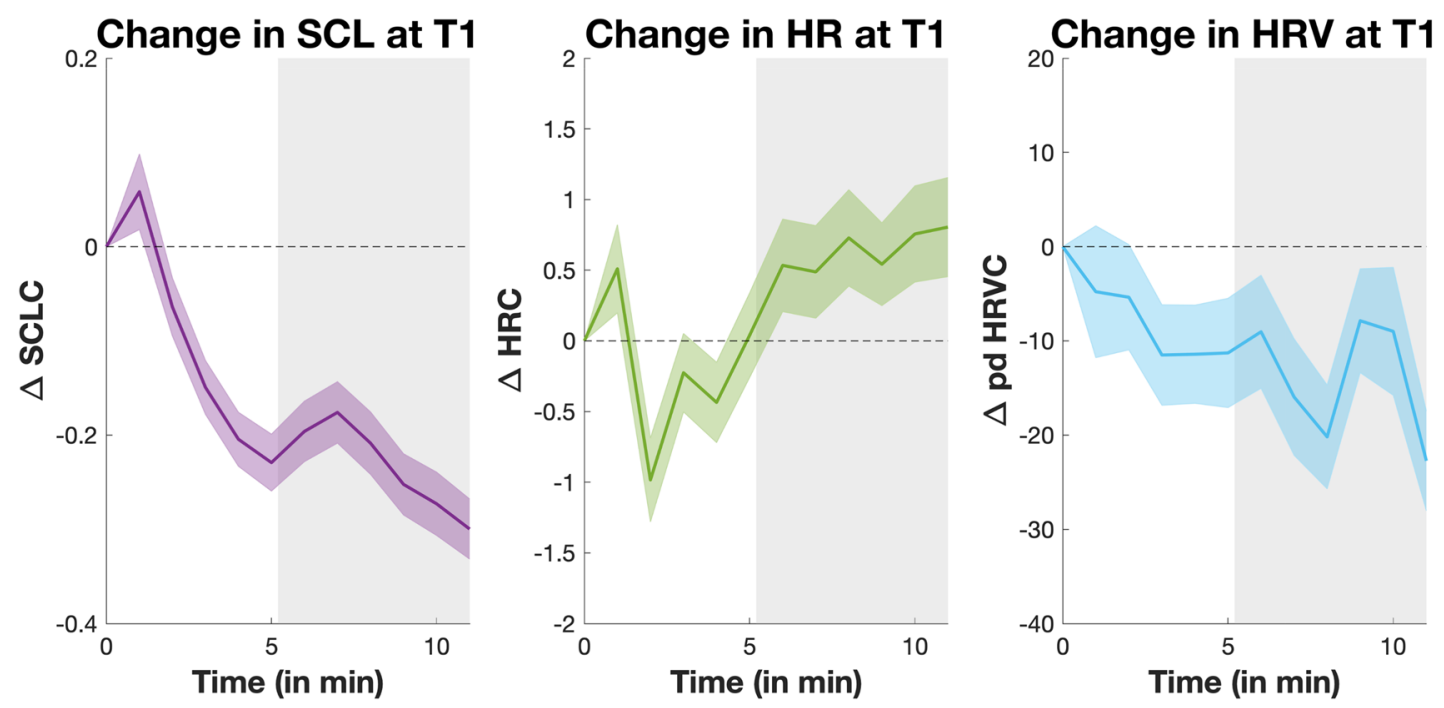
4Fig. 2 A Experimental procedure in sequential order and accompanied by approximate timings. Note. The data of the cognitive task and from the EEG recordings are not presented in this paper. B-F LKMS-related changes in self-report and physiological responses across groups pre-intervention. Error bars $= \pm 1$ SE. Shades represent \pm 1 SE. The grey-shaded area marks the time when participants focused on the self

correlations. Residualised gain scores, as a validated index of pre-post-treatment changes that controls for variance in initial pre-scores, were calculated for each person and change parameter by regression with postscore as outcome (Mintz et al., 1979; Williams et al., 1984). To account for multiple testing, we adjusted the $p$ value for number of tests.

\section{Sensitivity, Specificity and Accuracy}

In order to investigate if the change in physiological responses to the LKM-S correctly classifies MBCT patients from untreated, we determined sensitivity, specificity and accuracy.

\section{Results}

\section{Pre-intervention Effects}

For the pre-intervention analyses, data were collapsed across intervention groups (see Fig. 2B-F for visualisation of the data). We found a medium effect size for enhanced selfreported self-compassion after the LKM-S $(t(49)=3.50$, $p=0.001,95 \%$ CI $[2.77,9.99], d=0.50)$, but only a small non-significant effect size for reduced self-reported selfcriticism, $t(49)=1.54, p=0.129,95 \%$ CI $[-9.89,1.29]$, $d=0.22$.

For the physiological responses to the LKM-S, we found a reduction in SCL during the LKM-S when focusing on a loved one $(t(45)=-3.92, p>0.001,95 \%$ CI $[-0.17,-0.06]$, $d=0.57)$ and the self, $t(45)=-7.15, p>0.001,95 \% \mathrm{CI}$ $[-0.30,-0.17], d=1.04$, suggesting the absence of a defence response. While focusing on others, HR did not change $(t(45)=-0.88, p>0.384,95 \%$ CI $[-0.72,0.28]$, $d=0.13$ ), but interestingly significantly increased when the focus changed to the self, $t(45)=2.22, p=0.031,95 \%$ CI $[0.06,1.22], d=0.33$, suggesting an increase in physiological arousal. Analyses on HRV changes revealed a trend for decreased HRV for the focus on others $(t(45)=-1.95$, $p=0.057,95 \%$ CI $[-18.02,0.28], d=0.28)$ while there was a significant medium-size decrease in HRV when focusing on the self, $t(45)=-2.80, p=0.007,95 \% \mathrm{CI}$ [-24.29, -3.97$], d=0.41$, suggesting a decrease in parasympathetic activation.

\section{Treatment-Related Changes in Depression Symptoms and Dispositional Self-Compassion and Self-Criticism (Manipulation Checks)}

MBCT was accompanied by significant reductions in symptoms of depression and dispositional self-compassion and self-criticism whereas no such changes were observed in the control group (see Fig. 3 and a detailed description of these statistics in the supplementary results).

\section{Treatment-Related Changes in State Self-Compassion and Self-Criticism}

Both groups demonstrated a significant increase in self-compassion after the LKM-S at both testing sessions. In contrast, we did not find reductions in self-criticism. Reflecting the dispositional changes in self-compassion and self-criticism reported above, we observed increased state self-compassion and reduced state self-criticism baseline scores in the MBCT group post-treatment (see supplementary Figure S2 and supplementary results for a detailed description of these statistics).

\section{Treatment-Related Changes in Physiological Arousal and Parasympathetic Activation}

A detailed description of the results of the focus (others vs. self) $\times$ time (first vs. second assessment) $\times$ group ANOVA can be found in the supplementary materials. In the following, we report results from the difference waves as indicator of MBCT-related change in physiological responses (e.g. post-change score-pre-change score as depicted in Fig. 4).

\section{SCL Effects}

The focus $\times$ group analyses revealed a main effect of group, $F(1,42)=7.86, p=0.008, \eta_{p}^{2}=0.158$, indicating that relative to the control group, the MBCT group demonstrated decreased SCL in response to the LKM-S post-treatment as compared to pre-treatment. No other effects reached significance.

\section{HR Effects}

Analyses revealed a significant group effect, $F(1,43)=10.66$, $p=0.002, \eta_{p}^{2}=0.199$, suggesting an MBCT-related decrease in HR in response to the LKM-S. There were no effects for focus and no group $\times$ focus interaction.

\section{HRV Effects}

The focus $\times$ group ANOVA revealed a significant effect of focus, $F(1,43)=5.41, p=0.025, \eta_{p}^{2}=0.112$, and group, 

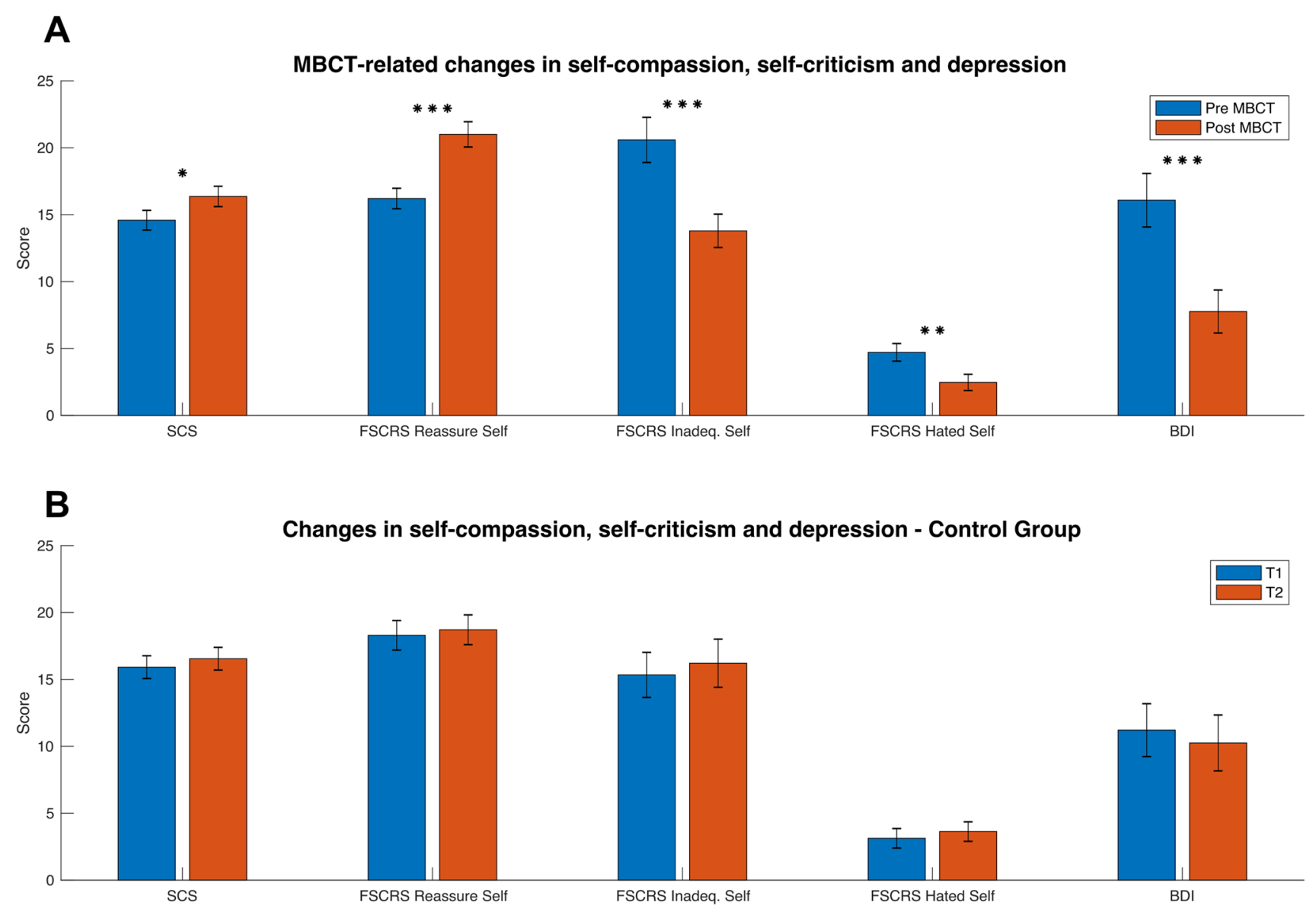

Fig. 3 MBCT-related changes in depression symptoms and dispositional self-compassion and self-criticism. Error bars $= \pm 1$ SE. Note: $*=p<.05 ; * *=p<.01 ; * * *=p<.001$ for $t$ tests comparing changes within each group

$F(1,43)=14.89, p<0.001, \eta^{2}=0.257$. These effects indicate higher HRV in response to the LKM-S when focusing on the self and generally higher MBCT-related HRV. In the first 3 min of self-focus, only the MBCT group showed mediumsize treatment-related elevation of HRV when changing the focus from others to the self (MBCT: $t(20)=2.20, p=0.040$, 95\% CI [1.29, 48.21], $d=0.48$; control: $t(23)=0.44$, $p=0.667,95 \% \mathrm{CI}[-7.81,11.99], d=0.09)$. This was supported by a trend for a focus $\times$ group interaction using this time window, $F(1,43)=3.76, p=0.059, \eta_{p}^{2}=0.080$.

\section{Associations Between Psychophysiological Change, Change in Dispositional Self and Symptom Reduction}

Within the whole sample, reductions in depression symptoms (BDI) were significantly positively correlated with reductions in self-criticism (FSCRS-inadequate self and hated self) with large correlation coefficients of $r=0.60$ (see supplementary Figure S4 for a detailed visualisation). Conversely, they were associated with an increases in self-compassion (SCS) and reassured self (FSCRS), effects ranging from medium $(r=-0.34)$ to large $(r=-0.53)$. Reductions in self-criticism (FSCRS-inadequate self) were significantly correlated with reductions in the heart rate and increases in the heart rate variability with absolute medium effects ranging from $r=0.32$ to $r=0.34$. Increases in selfreassurance (FSCRS) were significantly correlated with reductions in skin conductance and heart rate with medium effects ranging from $r=-0.31$ to $r=-0.32$.

Within the MBCT group (see supplementary Figure S4 for a detailed visualisation), reduction in skin conductance when directing compassion for others and for self was significantly correlated with BDI symptom reduction with medium correlation coefficients ranging from $r=0.46$ to $r=0.47$. Similarly, reductions in self-criticism (FSCRSinadequate self) were descriptively associated with treatment-related increases in heart rate variability when directing compassion to others and the self.

In addition, we found that change in HRV when directing compassion to the self correctly classified $76 \%$ of participants as MBCT participants with a sensitivity of 69.2 

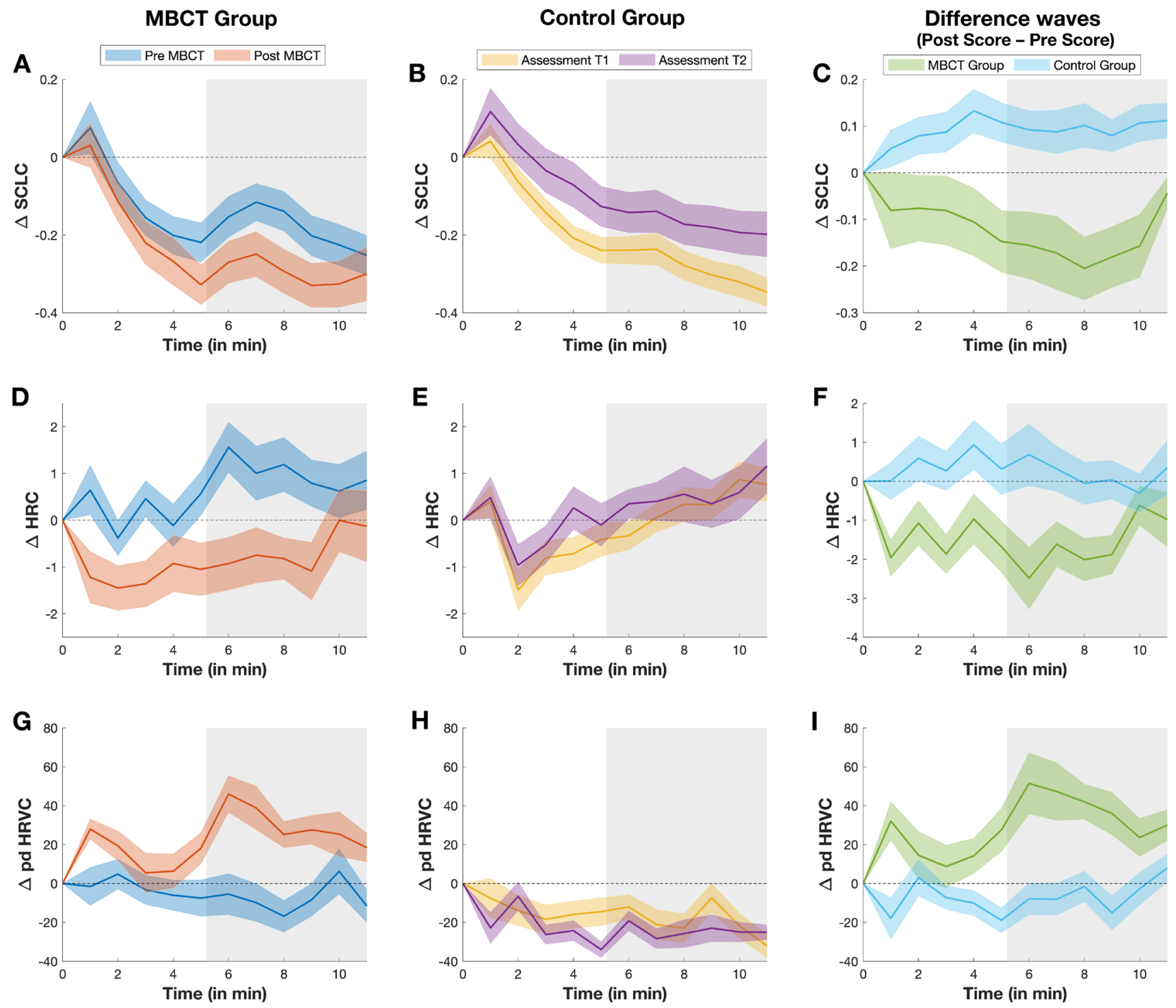

Fig. 4 MBCT-related changes in physiological arousal and parasympathetic activation. Note: Shades represent \pm 1 SE. The grey-shaded area marks the time when the participants focused on the self

and a specificity of 84.2 suggesting that 7 out of $10 \mathrm{MBCT}$ participants but only 2 out of 10 non-MBCT patients showed an increase in HRV at their second assessment when they direct compassion to themselves.

\section{Discussion}

By concomitantly assessing self-report and physiological responses to a self-compassion meditation, this study provides novel insights into positive self-referential processing as a potential marker and treatment target for recurrent depression. In our self-compassion meditation, individuals directed kind and self-affirming thoughts first to a significant other and then, for several minutes, to themselves
(Kirschner et al., 2019). We studied self-report and physiological responses to both phases of the self-compassion meditation as proxies for strategic or automatic self-referential processing respectively in currently remitted individuals with a history of three or more depressive episodes before and after they completed either an 8-week MBCT course or a waiting phase. Post-MBCT, we found evidence for higher meditation-induced strategic and automatic positive self and reduced negative self, whereas prior to treatment, these changes were only partially observed.

Our results not only replicated previous reports identifying self-reported changes in self-compassion and self-criticism through MBCT (Feldman \& Kuyken, 2011; Kuyken et al., 2010; van der Velden et al., 2015), but also extended this research in several ways. First, by showing reduced 
self-criticism and physiological arousal and increased selfcompassion and increased heart rate variability to a selfcompassion meditation after MBCT, we indicate that this intervention has the potential to reduce negative self-bias and to facilitate the psychophysiological benefits associated with a more positive self. This conclusion is supported by two observations. MBCT normalised physiological responses to the self-compassion exercise in individuals with recurrent depression towards a response pattern previously seen in healthy individuals (Kirschner et al., 2019; Rockliff et al., 2008) and associated with adaptive responses to distress (Arch et al., 2014) and psychological and physiological health (Thayer \& Lane, 2000, 2007). Additionally, our finding that the pre-to-post HRV increases when individuals direct compassion to themselves correctly classified individuals as having undergone MBCT with an accuracy of nearly $80 \%$ may suggest this as a potential biomarker to target in future research not only for improvements in emotion regulation (Thayer \& Lane, 2000) following MBCT but specifically of an improved positive self (Kirschner et al., 2019). These results contribute to existing evidence that negative self-bias in depression can be changed through therapies (Hilimire et al., 2015; Yoshimura et al., 2014, 2017) but critically extend this research by suggesting that MBCT may be uniquely placed to facilitate a more habitual, embodied positive self (Feldman \& Kuyken, 2011) by its focus on practising awareness and non-reactivity thus enabling patients to consciously choose thoughts, emotions and sensations (Chambers et al., 2009) with an orientation of acceptance. MBCT is therefore not only a cost-effective treatment alternative (Kuyken et al., 2015) but effectively addressing a key vulnerability for depression (Beck, 2008; Gotlib \& Joormann, 2010; Mathews \& MacLeod, 2005). This conclusion is also supported by emerging neural data (Farb et al., 2007) showing how mindfulness meditation is associated with experience-dependent plasticity in brain circuitries underlying self-referential processing (Lin et al., 2018).

Second, our findings shed light on the debate about strategic versus automatic negative self-bias in depression. The prior-to-the-intervention-phase transiently increased selfcompassion at the end of the self-compassion meditation can be seen as partial support for a temporarily increased positive self in remitted depressed patients. However, there was no reduction in state self-criticism suggesting persistence of negative self-bias beyond symptom reduction. In addition, the self-reported increase in self-compassion was not accompanied by the pattern of reduced physiological arousal and increased heart rate variability that we previously identified in healthy individuals (Kirschner et al., 2019). On the contrary, in those currently remitted but still at risk for depressive relapse, we found a significant increase in HR and decrease in HRV when participants focused on the self during the self-compassion meditation. This discrepancy may suggest that when remitted, individuals may be able to access less negative and more positive self at strategic but not automatic, habitual levels, thus leaving individuals vulnerable for relapse. This has been previously suggested by Shestyuk and Deldin (2010) who identified a latent neural vulnerability by studying early and late brain potentials to positive or negative self-referential adjectives. Individuals after CBT-induced remission from major depression showed brain electrical responses suggestive of a successfully reduced strategic but not automatic negative self-bias. Our finding of discrepant automatic and strategic self-referential processing prior to MBCT adds to the growing evidence that it is the habitual negative self-bias uniquely contributing to depressive relapse (LeMoult et al., 2017).

Third, our data suggest that without MBCT, automatic negative self-bias persists in people vulnerable to depression. This was evidenced by the absence of changes in dispositional self-compassion and self-criticism in the untreated remitted depressed patients. Moreover, this group did not only not show a normalisation of their physiological response pattern but, upon the second exposure to the selfcompassion meditation, they appeared to experience a more adverse reaction as indicated by significantly increased skin conductance responses from pre- to post-intervention phase. These findings support that directing compassion to the self is challenging for individuals with depression (Gilbert et al., 2014; Rockliff et al., 2008) and support our conclusion that aberrant psychophysiological responses to a self-compassion meditation are indicators of a persistent negative self-bias and reduced automatic positive self-representation.

Our results have important theoretical implications. Differential psychophysiological responses to a self-compassion meditation in line with MBCT-treatment status and the ability to tap into automatic self-referential processing suggest that our paradigm may lend itself for studying biomarkers of vulnerabilities for recurrent depression and successful treatment response. If replicated in future research, our paradigm may provide an easy-to-administer and cost-effective alternative to other more expensive or labour-intensive neuroscience approaches. Our paradigm may also help in understanding the time course of functional changes associated with symptom reduction and treatment. Whereas a one-offself-compassion meditation appeared to transiently induce a more positive self at strategic, top-down control level but no significant reduction in negative self at both self-report and physiological arousal level, our MBCT-related changes in comparison to a no-treatment control suggest an emerging transition towards a more automatic, habitual positive 
self-referential processing. Of interest was that we found the MBCT-related physiological changes to be associated with change in dispositional self and depressive symptom reduction. Positioning our findings within suggested brain plasticity processes associated with mindfulness meditation (Tang et al., 2015), our effects may represent short- to medium-term plasticity where bottom-up habitual processing starts to emerge in a more stable pattern that now needs to be maintained by regular practice which should activate these networks and thus lead to long-term functional plasticity (Bullmore \& Sporns, 2009) as observed in long-term meditators (Tang et al., 2015).

\section{Limitations and Future Research}

Our study has some limitations. First, although our experimental results were significantly associated with changes in validated psychometric instruments to assess dispositional self-compassion and self-criticism, we have not yet crossvalidated our paradigm with other typically applied measures for assessing negative self-bias such as self-referential tasks (Markus, 1977) or neural network analyses (Bullmore $\&$ Sporns, 2009). Future studies should therefore use an array of different measures and paradigms that have been shown to tap into automatic and strategic self-referential processing. In particular, to support our suggestion that we have identified a potential biomarker for a latent vulnerability in recurrent depression, it would be helpful to study the underlying neural correlates of our paradigm. This would allow us to compare them with the so-far identified neuro-signatures of self-referential processing associated with depression and its treatment and confirm if our markers lend themselves as an easy-to-administer proxy for brain plasticity.

Second, this study was conducted in a real-world clinical setting, making our findings susceptible to sampling bias. It should be noted that our sample was descriptively unbalanced on depressive symptoms and the inadequate self subscale of the FSCRS at baseline. When controlling for these confounds, the main treatment-related effects remain qualitatively unchanged (see supplementary results). Moreover, the core focus of this study was not to establish the effectiveness of MBCT, but to explore potential mechanisms of MBCT-related changes.

Third, the study did not use an active treatment control group preventing us from directly testing our claim that MBCT may be uniquely addressing automatic, habitual selfreferential processing in depression. Future research might address this by using an active control group like CBT or more MBCT-specific comparisons such as relaxation training or pseudo-meditations (Noone \& Hogan, 2018).

Fourth, this study did not include follow-up data which would have allowed examining whether changes in strategic and automatic self-bias as identified in our study predict reduced relapse rates and wellbeing at 1- or 2-year followup. Future studies should therefore include follow-ups for a better understanding of long-term, adaptive functional and structural plasticity associated with successful relapse prevention for depression and with the study of treatmentrelated effects.

Supplementary Information The online version contains supplementary material available at https://doi.org/10.1007/s12671-022-01845-3.

Acknowledgements This research was supported by a University of Exeter Graduate Fellowship that was awarded to H.K. and matchfunded to a Welcome Capital Bit Award to the Mood Disorders Centre, University of Exeter. We would first like to thank all our participants who took part in this research for their generosity and commitment. We are also extremely grateful to the therapists at the Mood Disorder Centre, Alison Evans, Jenny Wilks, Jerry Fox, and Jo Gosling, for delivering the MBCT courses and their help in recruitment. Finally, the authors thank Pia Pechtel for helpful discussions on the paper and proofreading.

Author Contribution HK: conceptualisation, methodology, writingoriginal draft preparation, formal analysis. WK: conceptualisation, methodology, writing-review and editing, supervision. AK: conceptualisation, methodology, writing — original draft preparation, formal analysis, supervision.

Funding Open Access funding enabled and organized by Projekt DEAL.

Data Availability The conditions of our informed consent form do not permit public archiving of the raw data because participants did not provide sufficient consent. Researchers who wish to access processed and anonymised data from a reasonable perspective should contact the corresponding author. Data will be released to researchers if it is possible under the terms of the General Data Protection Regulation (GDPR). All materials, processing and analysis scripts are available from the authors upon reasonable request.

\section{Declarations}

Ethics Approval The study protocol was approved by the school of psychology, University of Exeter ethics committee and the South West Cornwall and Plymouth NHS Research Ethics Committee.

Informed Consent Statement All participants provided written informed consent.

Conflict of Interest W.K. is the director of the Oxford Mindfulness Centre. W.K. receives payment for training workshops and presentations related to MBCT and donates all such income to the Oxford Mindfulness Foundation, a charitable trust that supports the work of the Oxford Mindfulness Centre. W.K. was, until 2015, an unpaid director of the Mindfulness Network Community Interest Company and gave evidence to the UK Mindfulness All Party Parliamentary Group. The remaining authors declared no additional potential conflicts of interest with respect to the authorship or the publication of this article. 
Open Access This article is licensed under a Creative Commons Attribution 4.0 International License, which permits use, sharing, adaptation, distribution and reproduction in any medium or format, as long as you give appropriate credit to the original author(s) and the source, provide a link to the Creative Commons licence, and indicate if changes were made. The images or other third party material in this article are included in the article's Creative Commons licence, unless indicated otherwise in a credit line to the material. If material is not included in the article's Creative Commons licence and your intended use is not permitted by statutory regulation or exceeds the permitted use, you will need to obtain permission directly from the copyright holder. To view a copy of this licence, visit http://creativecommons.org/licenses/by/4.0/.

\section{References}

American Psychiatric Association. (2013). Diagnostic and statistical manual of mental disorders. Author. https://doi.org/10.1176/appi. books. 9780890425596

Arch, J. J., Brown, K. W., Dean, D. J., Landy, L. N., Brown, K. D., \& Laudenslager, M. L. (2014). Self-compassion training modulates alpha-amylase, heart rate variability, and subjective responses to social evaluative threat in women. Psychoneuroendocrinology, 42, 49-58. https://doi.org/10.1016/j.psyneuen.2013.12.018

Beck, A. T. (2008). The evolution of the cognitive model of depression and its neurobiological correlates. American Journal of Psychiatry, 165(8), 969-977. https://doi.org/10.1176/appi.ajp.2008. 08050721

Beck, A. T., Steer, R. A., \& Brown, G. K. (1996). Manual for the BDI-II. Psychological Corporation. https://doi.org/10.1007/9781-4419-1005-9_441

Berntson, G. G., Bigger, J. T., Eckberg, D. L., Grossman, P., Kaufmann, P. G., Malik, M., Nagaraja, H. N., Porges, S. W., Saul, J. P., Stone, P. H., \& VanderMolen, M. W. (1997). Heart rate variability: Origins, methods, and interpretive caveats. Psychophysiology, 34(6), 623-648. https://doi.org/10.1111/j.1469-8986. 1997.tb02140.x

Bullmore, E., \& Sporns, O. (2009). Complex brain networks: Graph theoretical analysis of structural and functional systems. Natur Review Neuroscience, 10(3), 186-198. https://doi.org/10.1038/ $\operatorname{nrn} 2575$

Chambers, R., Gullone, E., \& Allen, N. B. (2009). Mindful emotion regulation: An integrative review. Clinical Psychology Review, 29(6), 560-572. https://doi.org/10.1016/j.cpr.2009.06.005

Diedrich, A., Grant, M., Hofmann, S. G., Hiller, W., \& Berking, M. (2014). Self-compassion as an emotion regulation strategy in major depressive disorder. Behaviour Research and Therapy, 58, 43-51. https://doi.org/10.1016/j.brat.2014.05.006

Disner, S. G., Shumake, J. D., \& Beevers, C. G. (2017). Self-referential schemas and attentional bias predict severity and naturalistic course of depression symptoms. Cognition \& Emotion, 31(4), 632-644. https://doi.org/10.1080/02699931.2016.1146123

Ellis, R. J., Sollers Iii, J. J., Edelstein, E. A., \& Thayer, J. F. (2008). Data transforms for spectral analyses of heart rate variability. Biomedical Sciences Instrumentation, 44, 392-397.

Farb, N. A., Segal, Z. V., Mayberg, H., Bean, J., McKeon, D., Fatima, Z., \& Anderson, A. K. (2007). Attending to the present: Mindfulness meditation reveals distinct neural modes of self-reference. Social Cognitive Affective Neuroscience, 2(4), 313-322. https:// doi.org/10.1093/scan/nsm030
Faul, F., Erdfelder, E., Lang, A. G., \& Buchner, A. (2007). G*Power 3: a flexible statistical power analysis program for the social, behavioral, and biomedical sciences. Behavior Research Methods, 39(2), 175-191. https://doi.org/10.3758/bf03193146

Feldman, C., \& Kuyken, W. (2011). Compassion in the landscape of suffering. Contemporary Buddhism, 12(1), 143-155. https://doi. org/10.1080/14639947.2011.564831

First, M. B., Spitzer, R., Gibbon, M., \& Williams, J. B. W. (1995). Structured clinical interview for DSM-IV Axis I disorder with psychotic screen. New York Psychiatric Institude. https://doi.org/ 10.1007/978-0-387-79948-3_2011

Gilbert, P. (2009). The compassionate mind. Constable.

Gilbert, P., Clarke, M., Hempel, S., Miles, J. N. V., \& Irons, C. (2004). Criticizing and reassuring oneself: An exploration of forms, styles and reasons in female students. British Journal of Clinical Psychology, 43, 31-50. https://doi.org/10.1348/014466504772812959

Gilbert, P., McEwan, K., Matos, M., \& Rivis, A. (2010). Fears of compassion: Development of three self-report measures. Psychology and Psychotherapy, 84(3), 239-255. https://doi.org/10.1348/ $147608310 \times 526511$

Gilbert, P., McEwan, K., Catarino, F., Baiao, R., \& Palmeira, L. (2014). Fears of happiness and compassion in relationship with depression, alexithymia, and attachment security in a depressed sample. British Journal of Clinical Psychology, 53(2), 228-244. https:// doi.org/10.1111/bjc.12037

Gotlib, I. H., \& Joormann, J. (2010). Cognition and depression: current status and future directions. In S. NolenHoeksema, T. D. Cannon, \& T. Widiger (Eds.), Annual review of clinical psychology (Vol. 6, pp. 285-312). https://doi.org/10.1146/annurev.clinpsy.121208. 131305

Hedlund, S., \& Rude, S. S. (1995). Evidence of latent depressive schemas in formerly depressed individuals. Journal of Abnormal Psychology, 104(3), 517-525. https://doi.org/10.1037/0021-843x. 104.3.517

Hilimire, M. R., Mayberg, H. S., Holtzheimer, P. E., Broadway, J. M., Parks, N. A., DeVylder, J. E., \& Corballis, P. M. (2015). Effects of subcallosal cingulate deep brain stimulation on negative self-bias in patients with treatment-resistant depression. Brain Stimulation, 8(2), 185-191. https://doi.org/10.1016/j.brs.2014.11.010

Holzel, B. K., Lazar, S. W., Gard, T., Schuman-Olivier, Z., Vago, D. R., \& Ott, U. (2011). How does mindfulness meditation work? Proposing mechanisms of action from a conceptual and neural perspective. Perspectives on Psychological Science, 6(6), 537-559. https://doi.org/10.1177/1745691611419671

Karl, A., Williams, M. J., Cardy, J., Kuyken, W., \& Crane, C. (2018). Dispositional self-compassion and responses to mood challenge in people at risk for depressive relapse/recurrence. Clinical Psychology \& Psychotherapy. https://doi.org/10.1002/cpp.2302

Kessing, L. V., Hansen, M. G., Kessing, L. V., Hansen, G. M., Andersen, G., \& Angst, J. (2004). The predictive effect of episodes on the risk of recurrence in depressive and bipolar disorders-a life-long perspective. Acta Psychiatrica Scandinavica, 109, 23-33. https://doi.org/10.1046/j.1600-0447.2003.00266.x

Kessler, R. C., Aguilar-Gaxiola, S., Alonso, J., Chatterji, S., Lee, S., Ormel, J., et al. (2009). The global burden of mental disorders: an update from the WHO world mental health (WMH) surveys. Epidemiologia e Psichiatria Sociale, 18, 23-33. https://doi.org/ 10.1017/s1121189x00001421

Kirschner, H., Kuyken, W., Wright, K., Roberts, H., Brejcha, C., \& Karl, A. (2019). Soothing your heart and feeling connected: A new experimental paradigm to study the benefits of self-compassion. Clinical Psychological Science, 7(3), 545-565. https://doi. org/10.1177/2167702618812438 
Kuyken, W., Watkins, E., Holden, E., White, K., Taylor, R. S., Byford, S., Evans, A., Radford, S., Teasdale, J. D., \& Dalgleish, T. (2010). How does mindfulness-based cognitive therapy work? Behaviour Research and Therapy, 48(11), 1105-1112. https://doi.org/10. 1016/j.brat.2010.08.003

Kuyken, W., Hayes, R., Barrett, B., Byng, R., Dalgleish, T., Kessler, D., Lewis, G., Watkins, E., Brejcha, C., Cardy, J., Causley, A., Cowderoy, S., Evans, A., Gradinger, F., Kaur, S., Lanham, P., Morant, N., Richards, J., Shah, P., ... Byford, S. (2015). Effectiveness and cost-effectiveness of mindfulness-based cognitive therapy compared with maintenance antidepressant treatment in the prevention of depressive relapse or recurrence (PREVENT): A randomised controlled trial. Lancet, 386(9988), 63-73. https:// doi.org/10.1016/s0140-6736(14)62222-4

Kuyken, W., Warren, F. C., Taylor, R. S., Whalley, B., Crane, C., Bondolfi, G., Hayes, R., Huijbers, M., Ma, H., Schweizer, S., Segal, Z., Speckens, A., Teasdale, J. D., Van Heeringen, K., Williams, M., Byford, S., Byng, R., \& Dalgleish, T. (2016). Efficacy of mindfulness-based cognitive therapy in prevention of depressive relapse: An individual patient data meta-analysis from randomized trials. JAMA Psychiatry, 73(6), 565-574. https://doi.org/10.1001/ jamapsychiatry.2016.0076

Lemogne, C., le Bastard, G., Mayberg, H., Volle, E., Bergouignan, L., Lehericy, S., Allilaire, J. F., \& Fossati, P. (2009). In search of the depressive self: Extended medial prefrontal network during selfreferential processing in major depression. Social Cognitive and Affective Neuroscience, 4(3), 305-312. https://doi.org/10.1093/ scan/nsp008

Lemogne, C., Mayberg, H., Bergouignan, L., Volle, E., Delaveau, P., Lehericy, S., Allilaire, J. F., \& Fossati, P. (2010). Self-referential processing and the prefrontal cortex over the course of depression: A pilot study. Journal of Affective Disorders, 124(1-2), 196-201. https://doi.org/10.1016/j.jad.2009.11.003

LeMoult, J., Kircanski, K., Prasad, G., \& Gotlib, I. H. (2017). Negative self-referential processing predicts the recurrence of major depressive episodes. Clinical Psychological Science, 5(1), 174-181. https://doi.org/10.1177/2167702616654898

Lin, Y. L., Callahan, C. P., \& Moser, J. S. (2018). A mind full of self: Self-referential processing as a mechanism underlying the therapeutic effects of mindfulness training on internalizing disorders. Neuroscience and Biobehavioral Reviews, 92, 172-186. https:// doi.org/10.1016/j.neubiorev.2018.06.007

Lykken, D. T., Rose, R., Luther, B., \& Maley, M. (1966). Correcting psychophysiological measures for individual differences in range. Psychological Bulletin, 66, 481-484. https://doi.org/10. 1037/h0023922

MacBeth, A., \& Gumley, A. (2012). Exploring compassion: A metaanalysis of the association between self-compassion and psychopathology. Clinical Psychology Review, 32(6), 545-552. https:// doi.org/10.1016/j.cpr.2012.06.003

Markus, H. (1977). Self-schemata and processing information about self. Journal of Personality and Social Psychology, 35(2), 63-78. https://doi.org/10.1037/0022-3514.35.2.63

Mathews, A., \& MacLeod, C. (2005). Cognitive vulnerability to emotional disorders. Annual Review of Clinical Psychology, 1, 167195. https://doi.org/10.1146/annurev.clinpsy.1.102803.143916

Mintz, J., Luborsky, P., \& Christoph, P. (1979). Measuring the outcomes of psychotherapy: Findings from the Penn Psychotherapy Project. Journal of Consulting and Clinical Psychology, 47(2), 319-334. https://doi.org/10.1037/0022-006X.47.2.319

National Institute for Health and Care Excellence [NICE]. (2010). Depression: the treatment and management of depression in adults (update). Author.
Neff, K. (2003a). Development and validation of a scale to measure self-compassion. Self and Identity, 2, 223-250. https://doi.org/10. $1080 / 15298860309027$

Neff, K. (2003b). Self-compassion: An alternative conceptualization of a healthy attitude toward oneself. Self and Identity, 2(2), 85-101. https://doi.org/10.1080/15298860390129863

Noone, C., \& Hogan, M. J. (2018). A randomised active-controlled trial to examine the effects of an online mindfulness intervention on executive control, critical thinking and key thinking dispositions in a university student sample. BMC Psychology, 6(1), 13. https:// doi.org/10.1186/s40359-018-0226-3

Piet, J., \& Hougaard, E. (2011). The effect of mindfulness-based cognitive therapy for prevention of relapse in recurrent major depressive disorder: A systematic review and meta-analysis. Clinical Psychology Review, 31, 1032-1040. https://doi.org/10.1016/j.cpr. 2011.05.002

Rockliff, H., Gilbert, P., McEwan, K., Lightman, S., \& Glover, D. (2008). A pilot exploration of heart rate variability and salivary cortisol respones to compassion-focused imagery. Jornal of Clinical Neuropsychiatry, 5, 132-139. https://psycnet.apa.org/record/ 2008-15384-002.

Rude, S. S., Wenzlaff, R. M., Gibbs, B., Vane, J., \& Whitney, T. (2002). Negative processing biases predict subsequent depressive symptoms. Cognition \& Emotion, 16(3), 423-440. https://doi.org/10. 1080/02699930143000554

Salzberg, S. (1997). Loving-kindness: the revolutionary art of happiness. Boston: Shambala.

Segal, Z., Williams, J. B., \& Teasdale, J. (2013). Mindfulness based cognitive therapy for depression: a new approach to preventing relapse (2nd ed.). Guildford Press.

Shestyuk, A. Y., \& Deldin, P. J. (2010). Automatic and strategic representation of the self in major depression: Trait and state abnormalities. American Journal of Psychiatry, 167(5), 536-544. https:// doi.org/10.1176/appi.ajp.2009.06091444

Sokolov, E. N. (1963). Perception and the conditioned reflex. Pergamon Press. https://doi.org/10.5694/j.1326-5377.1965.tb18452.x

Strauss, C., Lever Taylor, B., Gu, J., Kuyken, W., Baer, R., Jones, F., \& Cavanagh, K. (2016). What is compassion and how can we measure it? A review of definitions and measures. Clinical Psychology Review, 47, 15-27. https://doi.org/10.1016/j.cpr.2016.05.004

Tang, Y. Y., Holzel, B. K., \& Posner, M. I. (2015). The neuroscience of mindfulness meditation. Nature Review Neuroscience, 16(4), 213-225. https://doi.org/10.1038/nrn3916

Thayer, J. F., \& Lane, R. D. (2000). A model of neurovisceral integration in emotion regulation and dysregulation. Journal of Affective Disorders, 61, 201-216. https://doi.org/10.1016/S0165-0327(00) 00338-4

Thayer, J. F., \& Lane, R. D. (2007). The role of vagal function in the risk for cardiovascular disease and mortality. Biological Psychology, 74(2), 224-242. https://doi.org/10.1016/j.biopsycho.2005. 11.013

Tickell, A., Ball, S., Bernard, P., Kuyken, W., Marx, R., Pack, S., Strauss, C., Sweeney, T., \& Crane, C. (2020). The effectiveness of mindfulness-based cognitive therapy (MBCT) in real-world healthcare services. Mindfulness, 11(2), 279-290. https://doi.org/ 10.1007/s12671-018-1087-9

van der Velden, A. M., Kuyken, W., Wattar, U., Crane, C., Pallesen, K. J., Dahlgaard, J., Fjorback, L. O., \& Piet, J. (2015). A systematic review of mechanisms of change in mindfulness-based cognitive therapy in the treatment of recurrent major depressive disorder. Clinical Psychology Review, 37, 26-39. https://doi.org/10.1016/j. cpr.2015.02.001

Williams, R. H., Zimmerman, J. M., Rich, J. M., \& Steed, J. M. (1984). Empirical estimates of the validity of four measures of change. 
Perceptual and Motor Skills, 58, 891-896. https://doi.org/10. 2466/pms.1984.58.3.891

Williams, J. M., Healy, D., Teasdale, J. D., White, W., \& Paykel, E. S. (1990). Dysfunctional attitudes and vulnerability to persistent depression. Psychological Medicine, 20(2), 375-381. https://doi. org/10.1017/S0033291700017694

Williams, M. J., Dalgleish, T., Karl, A., \& Kuyken, W. (2014). Examining the factor structures of the five facet mindfulness questionnaire and the self-compassion scale. Psychological Assessment, 26(2), 407-418. https://doi.org/10.1037/a0035566

World Health Organization. (2008). The global burden of disease: 2004 update. WHO.

Yoshimura, S., Okamoto, Y., Onoda, K., Matsunaga, M., Okada, G., Kunisato, Y., Yoshino, A., Ueda, K., Suzuki, S., \& Yamawaki, S. (2014). Cognitive behavioral therapy for depression changes medial prefrontal and ventral anterior cingulate cortex activity associated with self-referential processing. Social Cognitive and Affective Neuroscience, 9(4), 487-493. https://doi.org/10.1093/ scan/nst009

Yoshimura, S., Okamoto, Y., Matsunaga, M., Onoda, K., Okada, G., Kunisato, Y., Yoshino, A., Ueda, K., Suzuki, S. I., \& Yamawaki, S. (2017). Cognitive behavioral therapy changes functional connectivity between medial prefrontal and anterior cingulate cortices. Journal of Affective Disorders, 208, 610-614. https://doi.org/ 10.1016/j.jad.2016.10.017

Publisher's Note Springer Nature remains neutral with regard to jurisdictional claims in published maps and institutional affiliations. 